\title{
Stratified Randomization
}

National Cancer Institute

\section{Source}

National Cancer Institute. Stratified Randomization. NCI Thesaurus. Code C147145.

A type of block randomization in which subjects are stratified into groups based on prognostic variables and then randomized into balanced treatment groups. 